\title{
Theoretical Study of Hole-Containing Macromolecular Diamond Lattices and Corona-Diamondoid Molecules or their Heteroatomic Derivatives ${ }^{\dagger}$
}

\author{
Alexandru T. Balaban \\ Texas A\&M University at Galveston, Department of Marine Sciences, 200 Seawolf Parkway, Galveston, TX 77553
}

\begin{abstract}
By analogy with benzenoids, diamond hydrocarbons (diamondoids for short) can be classified according to their dualists into catamantanes with acyclic dualists, perimantanes with dualists having 6-membered rings, and coronamantanes having larger rings that are not peripheries of 6-membered ring aggregates. Coronoid diamond hydrocarbons may be either tridimensional portions of the macromolecular diamond lattice with holes examined in an earlier article and here, or hollow smaller molecules (coronamantanes) also discussed in the present paper, with 3D or quasi-2D holes. In the case of lattices, the effect of external hydrogen atoms is negligible but it cannot be ignored in the case of coronamantanes. Both the outer and inner dangling bonds may be connected with hydrogen atoms, or some of the inner carbon atoms with dangling bonds may be replaced by heteroatoms $(\mathrm{N}, \mathrm{O}$, or $\mathrm{S}$ ) which might be able to bind metallic cations with coordinative bonds. A convenient means of structure representation uses dualists of the diamondoids. The holes may be isolated as are "closed pores" in polymer foams, or they may communicate forming tunnels as for 'open pores' in 'sponge foams'. A special discussion is reserved for coronamantane analogs of the quasi-planar coronoids. In these cases the diamondoid is quasi-flat and the quasi-2D hole or tunnel is accessible on both sides; it may be chiral or achiral. The diamondoid analog of kekulene is a cyclohexadecamantane $\mathrm{C}_{64} \mathrm{H}_{64}$, a challenge for the future. (doi: 10.5562/cca2288)
\end{abstract}

Keywords: diamondoids, coronamantanes, coronoids, dualists

\section{INTRODUCTION}

In a preceding article published in $1996,{ }^{1}$ holes in diamond or carbon nitride lattices were examined theoretically. Only macromolecular lattices were discussed, implying systems involving billions of billions of atoms, in lattices that were constitutionally similar but not necessarily identical, perhaps differing in molecular weights. Thus peripheral effects were considered to be negligible, and the discussion could be concentrated on the innards of these lattices. On removing atoms from the diamond lattice in order to create holes, the remaining carbon atoms with dangling bonds were considered to be replaceable by heteroatoms such as nitrogen (hence converting the diamond lattice into a carbon nitride lattice). Depending on the type of holes, they were analogs of foams with isolated holes (closed pores) or with communicating holes (open pores) yielding channels or tunnels in the lattice. Metallic cations could then form coordinative bonds with heteroatoms, whereas compensating electrical charges could either be provided by anions in the available spaces/channels, or by further replacement of carbon atoms by boron atoms.

In the present paper, a different theoretical angle is considered, again starting from the diamond lattice, but now dividing this lattice into smaller (molecular) fragments, with peripheral carbon atoms connected to hydrogen atoms, resulting in what are called diamondoids or diamond hydrocarbons. Now holes in diamondoids can be theoretically examined and the inner carbon atoms with dangling bonds may either be connected to hydrogen atoms or be replaced by hetero-atoms such as nitrogen (replacing tertiary $\mathrm{CH}$ groups, and $\mathrm{O}$ or $\mathrm{S}$ heteroatoms when replacing secondary $\mathrm{CH}_{2}$ groups). Again, if coordinative bonds to metallic cations are to be considered, then the compensating negative charges have also to be somehow included into the picture. Such substances contain billions of billions of identical molecules.

First for analogy and comparison purposes, the 2-dimensional coronafusenes with benzenoid rings and also holes between them will be briefly discussed. Then

\footnotetext{
$\dagger$ Dedicated with gratitude to Doug Klein's $70^{\text {th }}$ anniversary.

* Author to whom correspondence should be addressed. (E-mail: balabana@tamug.edu)
} 
diamondoids with holes will be considered (coronamantanes), starting with discussing systems that have their holes completely surrounded by carbon atoms in 3D structures, and subsequently with holes accessible from both sides in quasi-2D structures, which are very similar to the planar coronafusenes.

\section{Coronaphenes or Coronafusenes (Structures with Holes within Polycyclic Benzenoids)}

The two carbon allotropes that have been known for a long time, graphite and diamond, contain $\mathrm{sp}^{2}-$ and $\mathrm{sp}^{3}$ hybridized carbon atoms, respectively. Transition metals $(\mathrm{Ni}, \mathrm{Pd}, \mathrm{Pt})$ in the presence of hydrogen have long been known to catalyze hydrogen addition to aromatic rings. In the 1970 s it was shown that $\mathrm{Ni}, \mathrm{Pd}, \mathrm{Pt}$, $\mathrm{Fe}, \mathrm{Co}$, or $\mathrm{Ag}$ nanoparticles can etch graphite in the presence of hydrogen, resulting in straight nanoscale channels that have intersecting angles of integer multiples of $30^{\circ}$, dictated by the anisotropic crystallographic 'zigzag' or 'armchair' directions of the honeycomb net. ${ }^{2}$ More recently these experiments have been extended to graphene sheets and to reductive or oxidative cleavage of CC bonds in graphite or graphene. ${ }^{3}$ A recent paper published in Nature Communications presents definite proof by scanning electron microscopy (SEM) and scanning tunneling microscopy (STM) for the carving of open channels and trenches or internal tunnels in graphite, advocating various uses of such nanoporous graphite as anode material for lithium batteries, or for biomedical applications. $^{4}$

Corona-condensed benzenoid hydrocarbons (coronaphenes, ${ }^{5}$ coronoids, or coronafusenes) have fused benzenoid rings surrounding larger rings. From the rich literature on this topic, only a few references are
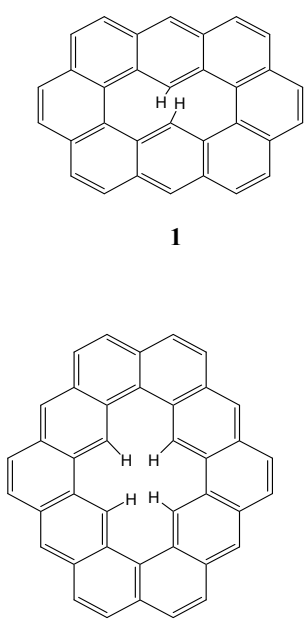

3

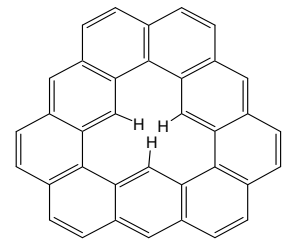

2

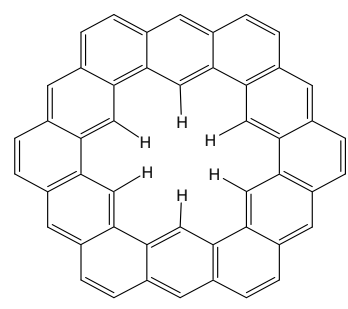

4
Figure 1. Coronafusenes $\mathbf{1}$ to $\mathbf{4}$ with $8,9,10$, and 12 benzenoid rings, respectively. selected. ${ }^{6-9}$ Klein with his coworkers published seminal papers on this topic,${ }^{10}$ and he reviewed defected graphitic nanostructures. ${ }^{11}$ The smallest coronoids such as kekulene (4) have just one "layer" or "rim" of condensed benzenoid rings. In the following we discuss only kekuléan coronoids, which have one or several Kekulé structures. By analogy with coronoids, which have "holes" inside a benzenoid (hosting interior hydrogen atoms), it will be interesting to define and study the corresponding hydrocarbons derived from the diamond lattice. In subsequent molecular structures of coronoids 1-4, outer hydrogen atoms will not be shown, and only inner hydrogens will be displayed.

The smallest kekuléan coronoid with 8 benzenoid rings (1) has a "hole" with two hydrogen atoms inside, replacing a deleted central $\mathrm{C}-\mathrm{C}$ bond in ovalene (circum-naphthalene) which has 10 benzenoid rings. Further, on deleting the central carbon atom of a nonkekuléan system with 12 benzenoid rings and an odd number of carbon atoms, the next higher coronoid (2) is obtained with three colliding hydrogens. Next, on deleting the central pair of adjacent carbon atoms in a benzenoid (circumpyrene) with 14 rings, one obtains the coronoid (3) having two pairs of colliding hydrogens. In this series the steric strain decreases gradually, but kekulene (4) prepared by Staab and Diederich is the first non-strained coronoid, with six inner hydrogens which are sufficiently remote from each other for minimizing any strain. ${ }^{12,13}$ For arriving at the kekulene structure, one has to excise the six carbon atoms of the central benzenoid ring from the kekuléan circumcoronene with 19 benzenoid rings.

A coronafusene with an even larger hole is shown in Figure 2. It has 14 benzenoid rings and may be considered to result from circum-ovalene by deleting the inner 10 carbon atoms of a naphthalene skeleton.

Katritzky and Marson attempted unsuccessfully to prepare a diazakekulene as a possible ligand for metallic cations via a related pyrylium bis(trifluoromethanesulfonate) shown in the right side of Figure 3, followed by treatment with ammonia in order to obtain the corresponding pyridine and then dehydrogenation for removal of twelve outward hydrogen atoms. Although the corresponding pyrylium salt was prepared, ${ }^{14}$ it could never be obtained pure.

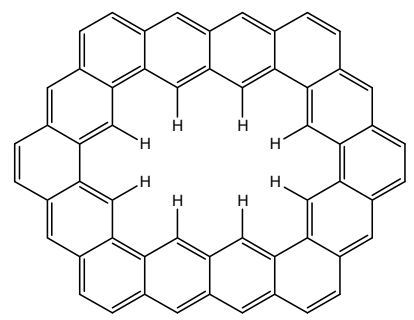

Figure 2. A coronafusene with 14 benzenoid rings. 

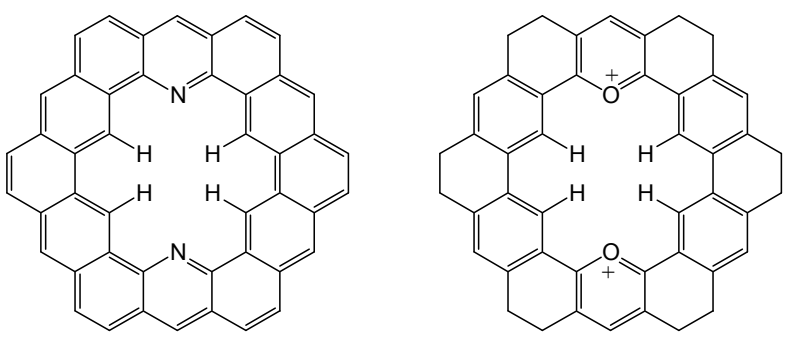

Figure 3. The hypothetical bis-azakekulene and on the right side the corresponding dodecahydro-bis-oxoniakekulene dication with two pyrylium rings.

\section{Coronamantanes Having Holes Completely Encased by Adamantane Units: 3D-Coronamantanes}

On joining together several benzenoid rings towards the graphene lattice, or building up adamantane units towards the diamond lattice, an essential difference appears between these two lattices. Unlike graphene which is a $2 \mathrm{D}$-lattice, ${ }^{15,16}$ diamond has a $3 \mathrm{D}$-lattice, and thus whenever two carbon atoms become situated at the single $\mathrm{C}-\mathrm{C}$ bond distance, this bond snaps into existence. By contrast, helicenes can be formed in the corresponding circumstance when one adds new benzenoid rings by evading into the $3^{\text {rd }}$ dimension; this can happen also with other planar nets, yielding for instance heliphenes with alternating six- and fourmembered rings. ${ }^{17}$

The present theoretical communication discusses a neglected detail in the related topic involving holes or tunnels in the other well-known carbon allotrope, diamond, the hardest known material. Nowadays along with the expensive natural diamond used for jewels, industrial synthetic diamonds are widely used for drill bits and saws, or as abrasive diamond powder. ${ }^{18}$ Chemical vapor deposition (CVD) has progressed to the extent that it may produce gem-quality diamonds, and only elaborate analytical methods and gemological tests may distinguish natural from CVD diamonds. ${ }^{19,20}$

For benzenoids and diamondoids, dualists consist of vertices placed at the center of each unit (benzenoid ring or adamantane cell) and edges connecting units sharing a CC bond or a chair-shaped ring, respectively. The code for diamondoid isomers (introduced by Balaban and Schleyer) is a succession of digits 1, 2, 3, or 4 for the directions of $\mathrm{CC}$ bonds around tetrahedral $\mathrm{C}$ atoms, between two ends of the molecule. ${ }^{21}$ When going from a planar benzenoid to a three-dimensional diamondoid, one has to take chirality into account:

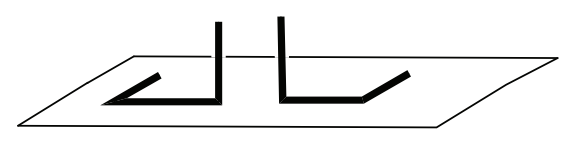

Figure 4. A pair of enantiomeric protochirons.
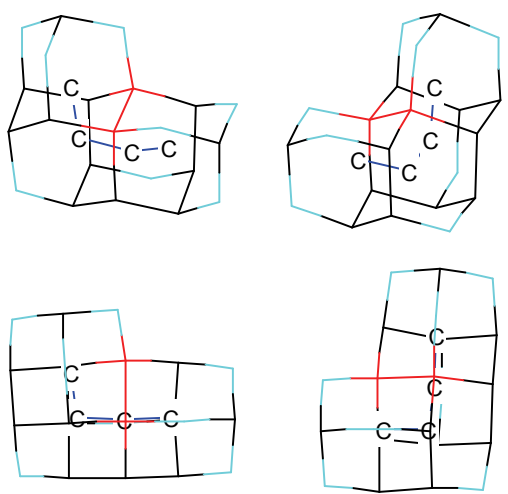

Figure 5. A pair of enantiomeric tetramantanes: upper row, side-view; lower row, view along a CC bond. In this and many subsequent figures the color code uses red for quaternary carbon atoms, black for tertiary carbons $(\mathrm{CH})$, light blue for secondary carbons $\left(\mathrm{CH}_{2}\right)$ and dark blue for the dualist represented by a chain of letters $\mathbf{C}$ at the Centers of adamantane Cells (units).

indeed, there one finds two enantiomers which may be encoded as [123]tetramantane and [124]tetramantane. Their dualists mimic the staggered gauche rotamers of $n$-butane; whereas these alkane rotamers are interconvertible with a very low rotation barrier, there is no such interconversion between the two enantiomeric tetramantanes. It was argued that the smallest element of asymmetry in chemistry is not an $\mathrm{sp}^{3}$-hybridized atom connected to four different ligands (a 5-vertex chemical graph), but a sequence of three non-planar covalent bonds ("protochirons") as depicted in Figure 4 with arbitrarily selected right angles (a 4-vertex chemical graph with $90^{\circ}$ angles)..$^{22,23}$

In Figure 5 one can see the two tetramantane enantiomers where the bond angles of the dualists are the tetrahedral angles of $109.5^{\circ}$.

To complete the picture, one should not forget that in addition to the two above-mentioned non-branched catafusenes, there is a third constitutional isomer of tetramantane, the branched [1(2)3]tetramantane which, like [121] tetramantane, is achiral.

In one of the next sections we will examine holes in a quasi-planar diamond lattice, whose dualist is "perhydrographene"; a portion of it is represented in Figure 3. We will call it "one-tier diamond lattice".
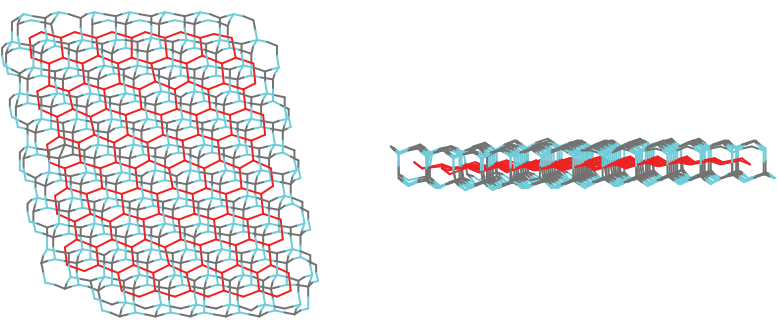

Figure 6. A portion of the one-tier diamond lattice: top-view (left) and side-view (right). 

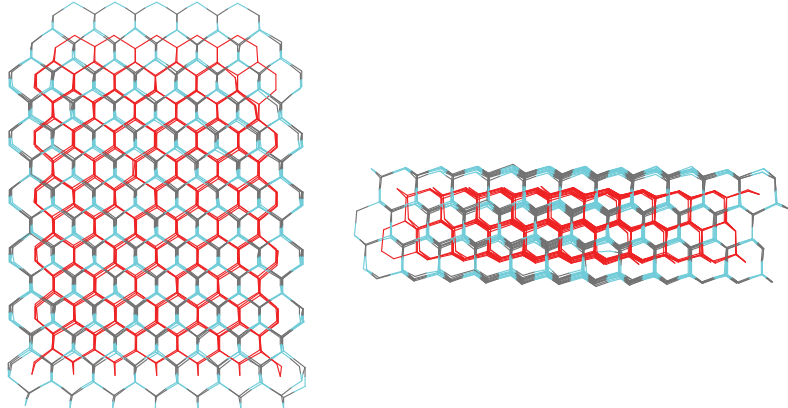

Figure 7. A portion of the three-tier diamond lattice: top-view (left) and side-view (right); color code as in Figure 6.

Since the diamond lattice is bipartite, the color code in Figure 6 is different, having all carbon atoms colored alternatively in blue and gray, and the dualist in red.

On considering more than one tier of quasi-planar adamantane units, one obtains a model of the real multitier diamond lattice. Figure 7 presents two views of a portion of the three-tier diamond lattice.

The diamond lattice can accommodate either quasi-2D or 3D holes in its structure. We shall discuss first the latter ones. In the following figures, hydrogens will be always shown as dangling bonds, unless otherwise stated.

If one deletes a $\mathrm{C}-\mathrm{C}$ bond from the diamond lattice and then one surrounds the "hole" with adamantane cells, then the "hole" will host two colliding hydrogens, similarly to the situation in $\mathbf{1}$. On deleting one carbon atom in the diamond lattice, the "hole" will now have four colliding hydrogens, by analogy with 2 . On deleting a pair of adjacent carbon atoms in the diamond lattice, the "hole" will have two pairs of hydrogen triplets, corresponding to the situation in $\mathbf{3}$. For obtaining the first non-strained 3D-coronamantane, one has to excise the ten carbon atoms of an adamantane cell. In the following, this non-strained 3D-coronamantane will be shown by means of several illustrations. Figures 8 and 9 are stereo-views after and before excision of the $\mathrm{C}_{10}$ cell, respectively.

Figure 10 displays the same situation without using stereo-views in more detail showing explicitly the 16 inner hydrogens connected to red-colored carbon
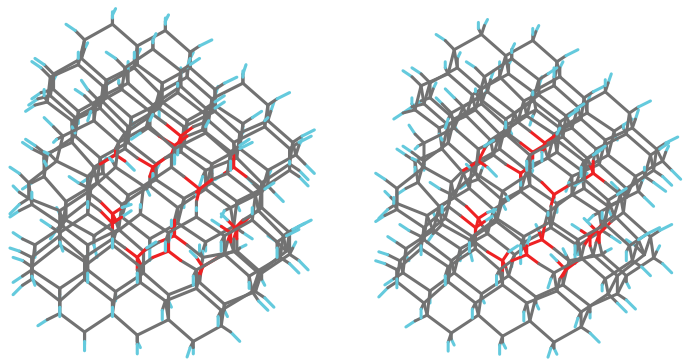

Figure 8. Diamond lattice with an "adamantane cell" hole bordered by vertices colored in red (stereo-view).
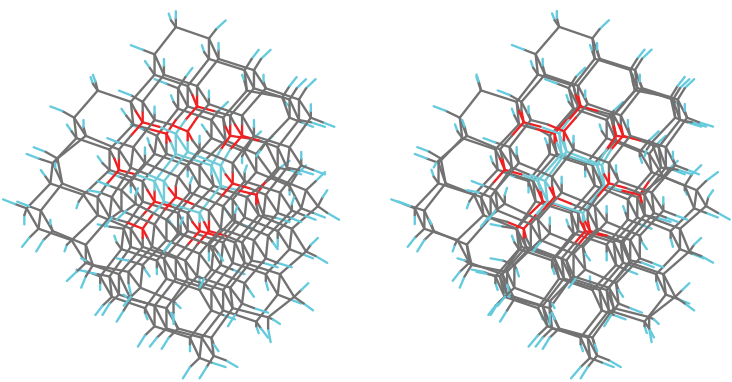

Figure 9. Diamond lattice with an "adamantane cell" colored blue bordered by vertices colored in red (stereo-view).

atoms (after excision of the $\mathrm{C}_{10}$ cell). Before the excision, the $\mathrm{C}_{10}$ adamantane cell which is going to be excised is shown colored in blue in Figure 11, with adjacent vertices colored in red.

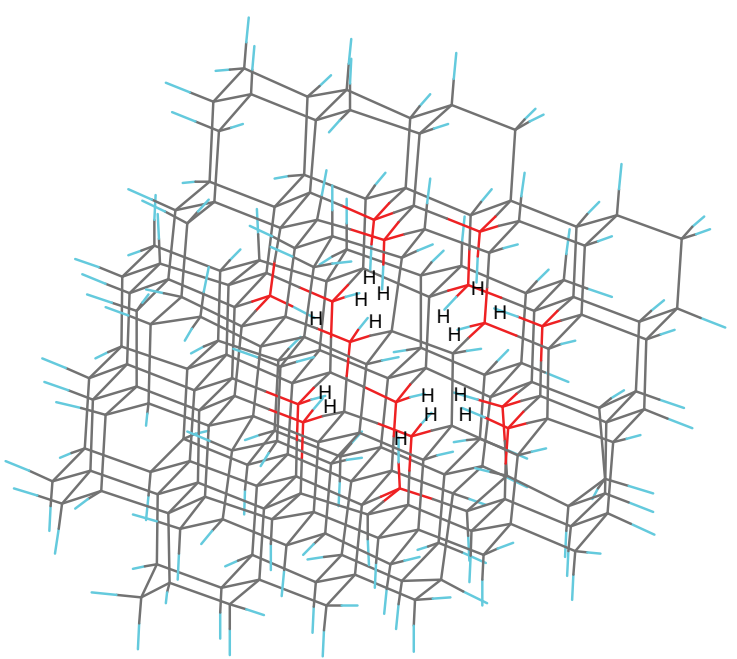

Figure 10. A 3D-coronamantane with a hole corresponding to an excised adamantane cell.

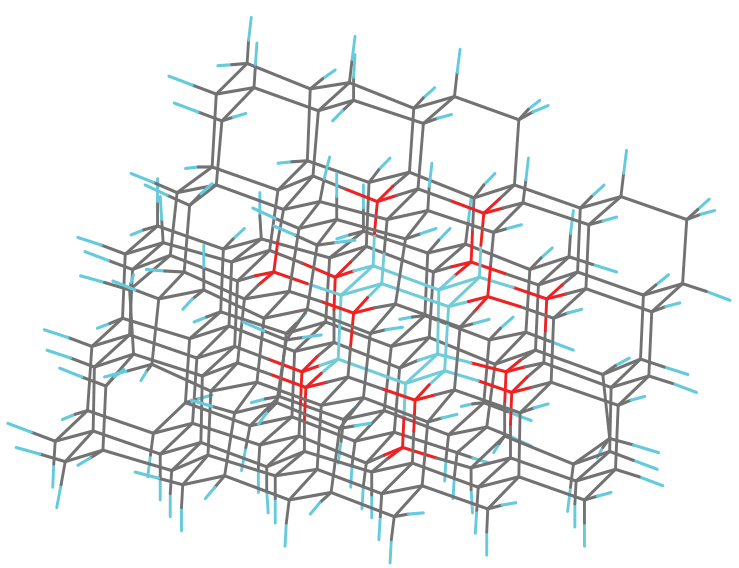

Figure 11. A portion of the diamond lattice with an adamantane cell colored in blue, and with red-colored carbon atoms adjacent to the carbons in the central adamantane cell. 


\section{Quasi-2D Coronamantanes with Open Holes or Tunnels}

A quasi-flat portion of the diamond lattice with peripheral hydrogens and its dualist (with an outer contour of 14 vertices, represented by red lines connecting centers of diamondoid units marked with letter $\mathrm{C}$, and devoid of hydrogen atoms) is shown in Figure 12. The dualist matches exactly the carbon skeleton of perhydropyrene. Similarly, the dualist of quasi-flat diamondoids matches exactly a portion of perhydrographene.

Following the example of kekulene (4) where one had to erase an inner benzenoid ring to yield a coronafusene (a benzenoid with a hole without conflicting inner hydrogens), it will be seen that a similar situation is encountered for coronamantanes. A 2D hole out of a quasi-flat diamond portion (a 2D-coronaman-tane) is shown in Figure 13. It has the global topology of a doughnut. The dualist of this 2D-coronamantane is the red line devoid of hydrogen atoms. As shown in this figure, there is no significant steric hindrance between the two pairs of inner hydrogens connected to the two secondary carbon atoms. From a quasi-flat portion of the diamond lattice, the hole inside this 2D-coronamantane with 18 adamantane units corresponds to having erased the adamantane units with their central six-membered ring in the dualist of that portion of the diamond lattice. An interesting ligand for metallic cations would result by replacing these two inner $\mathrm{CH}_{2}$ groups by oxygen or sulfur heteroatoms.

By analogy with benzenoids, diamondoids can be classified according to their dualists into catamantanes with acyclic dualists, perimantanes with dualists having 6-membered rings, and coronamantanes having larger rings that are not peripheries of 6-membered ring aggregates. Both regular and irregular $[n]$ catamantanes with $n$ adamantane units (cells) have acyclic dualists. Molecular formulas of regular $[n]$ catamantanes are $\mathrm{C}_{4 n+6} \mathrm{H}_{4 n+12}$; when there are fewer $\mathrm{C}$ or $\mathrm{H}$ atoms for a given $n$, the

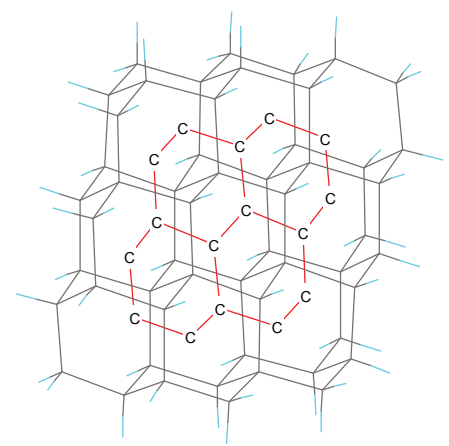

Figure 12. A small portion of a quasi-flat layer of adamantane units from the diamond lattice, with the corresponding dualist (red lines linking centers of adamantane units indicated by letter C).
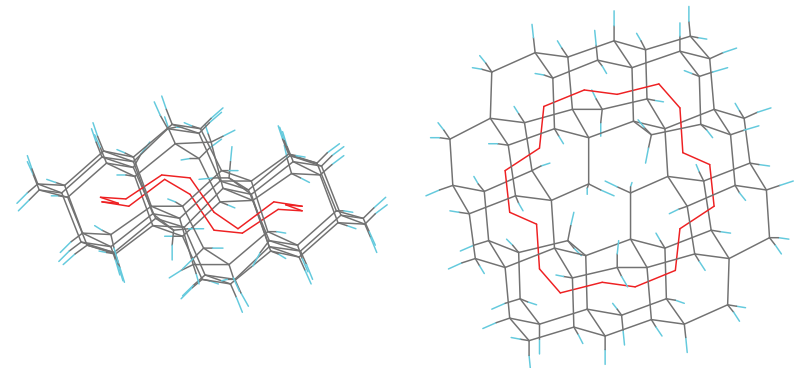

Figure 13. Side and front views of a 2D-coronamantane with 18 adamantane units. The red line connecting the centers of these units is the dualist.

diamondoid is either an irregular catamantane or a perimantane, according to the acyclic or cyclic dualist, respectively. Codes describing $[n]$ catamantane constitutions use $n-1$ digits (from 1 to 4 ), indicating the four tetrahedral directions of the $n-1$ dualist edges. Their dualists mimic staggered alkane conformers. $[n]$ Perimantanes have six-membered rings (chair-shaped hexagons), and for monocyclic systems with $n$ adamantne units, their codes need not indicate all directions of the $n$ edges, because $n-1$ digits suffice since the last $\left(n^{\text {th }}\right)$ edge is automatically closed when its two endpoints reach the $\mathrm{C}-\mathrm{C}$ bonding distance. A similar situation occurs also with $2 \mathrm{D}[n]$ coronamantanes so that the dualist of [18]coronamantane from Figure 13 has the code 12123231312123231 with only 17 digits. An even larger hole of a coronamantane with 22 adamantane units, again with insignificant steric interference of the four pairs of inner hydrogens, is shown in Figure 14. Its code is 121213132321212131323 . If the corresponding four $\mathrm{CH}_{2}$ groups would be replaced by $\mathrm{O}$ or $\mathrm{S}$ heteroatoms, another ligand for different metallic cations would be the result. One can see that dualists of quasi-2D structures contain only digits 1, 2, and 3 (and not 4).

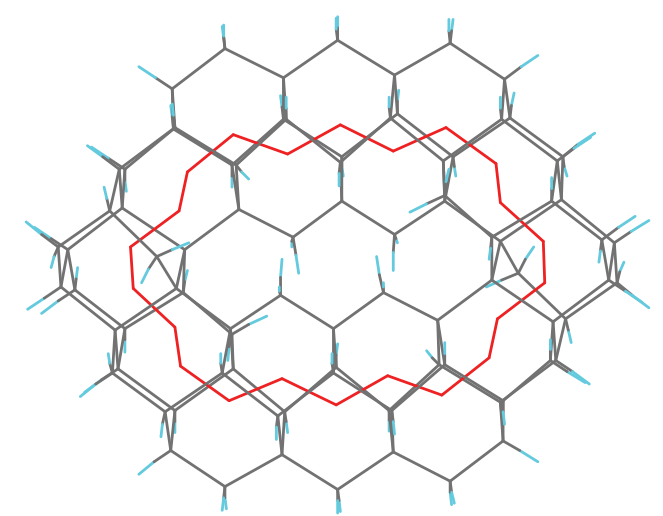

Figure 14. A quasi-flat coronamantane with a dualist having 22 vertices. The red line is the dualist. 


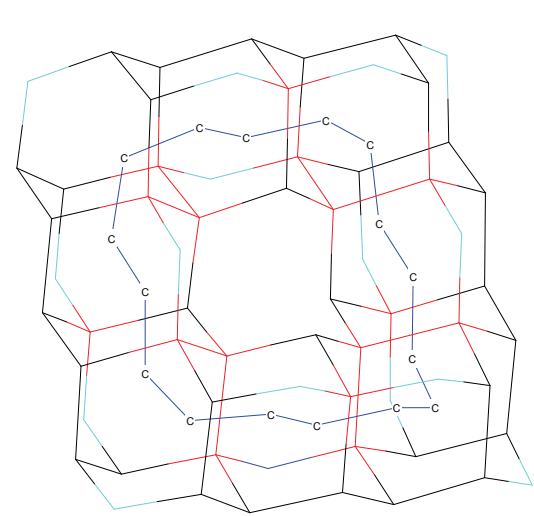

Figure 15. The diamondoid $\mathrm{C}_{64} \mathrm{H}_{64}$ analog of kekulene; quaternary carbons are red, tertiary $\mathrm{CH}$ groups are black, and secondary $\mathrm{CH}_{2}$ groups are light blue. The dualist is dark blue and its vertices are denoted by $\mathrm{C}$.

A challenge for synthetic chemists or for those hoping to isolate diamondoids from petroleum is the analog of kekulene: [1212343421214343]cyclohexadecamantane, a beautiful molecule shown in Figure 15. Being constituted of 16 adamantane units, it has point group $C_{2 v}$. Its molecular formula is $\mathrm{C}_{64} \mathrm{H}_{64}$, and its partitioned formula (according to quaternary, tertiary, and secondary carbon atoms $)^{24,25}$ is $\mathrm{C}_{16}(\mathrm{CH})_{32}\left(\mathrm{CH}_{2}\right)_{16}$. Two pairs of hydrogen atoms of the central eight-membered ring may cause a slight steric strain.

\section{Achiral and Chiral Coronamantanes}

On looking at the lower row in Figure 5, it can be seen that the diamond lattice can be projected on a square lattice if one looks along a CC bond. Then the "kink" of the chiral [123]triamantane appears as an angle of $90^{\circ}$. With four such "kinks" at the corners of a square corresponding to four [12] triamantane units, one obtains the coronamantane represented in Figure 16. It has the molecular formula $\mathrm{C}_{80} \mathrm{H}_{80}$, and partition formula $\mathrm{C}_{20}(\mathrm{CH})_{40}\left(\mathrm{CH}_{2}\right)_{20}$. For simplicity, we will denote it as "achiral 6666" indicating that each of the four sides of the projected square contains six vertices of the dualist.

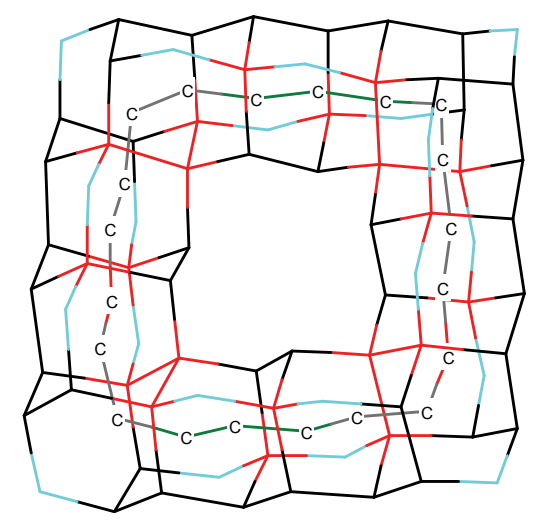

Figure 16. The "achiral 6666" coronamantane.
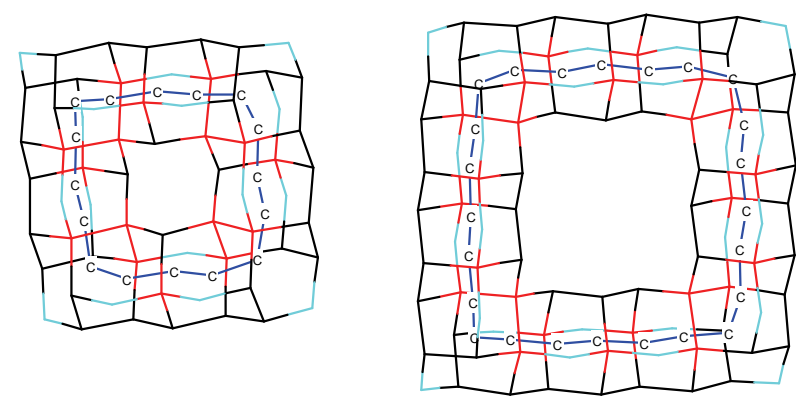

Figure 17. Achiral coronamantane-5555 (left) and coronamantane-7777 (right).

In this case there are four inner hydrogen atoms in the "hole" of the coronamantane that interfere sterically. It has mirror-plane symmetry. This coronamantane can be considered as the result of cutting off one pair of adjacent carbon atoms with all their attachments from the one-tier diamond lattice, and of leaving only the closest 20 adamantane units, with the circular dualist.

Figure 17 presents achiral coronamantanes denoted conventionally as 5555 and 7777, respectively. Their molecular formulas are $\mathrm{C}_{64} \mathrm{H}_{64}$ and $\mathrm{C}_{96} \mathrm{H}_{96}$, respectively, whereas their partitioned formulas are $\mathrm{C}_{16}(\mathrm{CH})_{32}\left(\mathrm{CH}_{2}\right)_{16}$ and $\mathrm{C}_{24}(\mathrm{CH})_{48}\left(\mathrm{CH}_{2}\right)_{24}$, respectively. The former can be considered as the result of removing one carbon atom with all its attachments from the one-tier diamond lattice, and of keeping only the closest adamantane units with their circular dualist of 16 vertices. It has severe steric interference of all four inner hydrogen atoms. The latter, without steric interference from inner hydrogens, can be viewed similarly as the result of removing a chair-shaped hexagon of carbon atoms with all their attachments.

An achiral coronamantane with a projected rectangle of vertices for the dualist denoted as 5757 is shown in Figure 18. Its molecular and partitioned formulas are $\mathrm{C}_{80} \mathrm{H}_{80}$ and $\mathrm{C}_{20}(\mathrm{CH})_{40}\left(\mathrm{CH}_{2}\right)_{20}$, respectively. It is noteworthy that these formulas are exactly the same as those for the achiral coronamantane-6666. It is the result of removing a $\mathrm{CCC}$ triplet from the one-tier diamond lattice, and has steric interference of six inner hydrogens connected to adjacent carbon atoms.

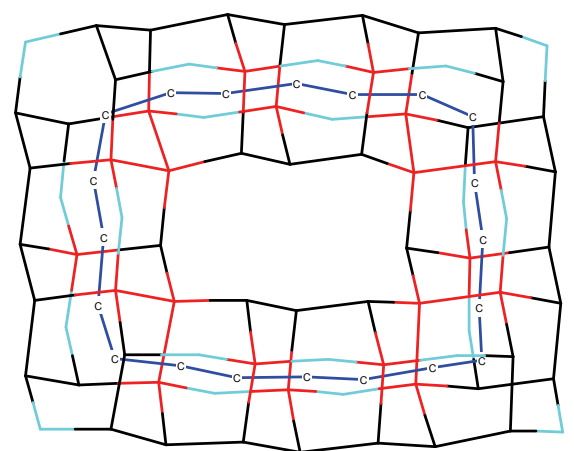

Figure 18. The achiral coronamantane-5757. 


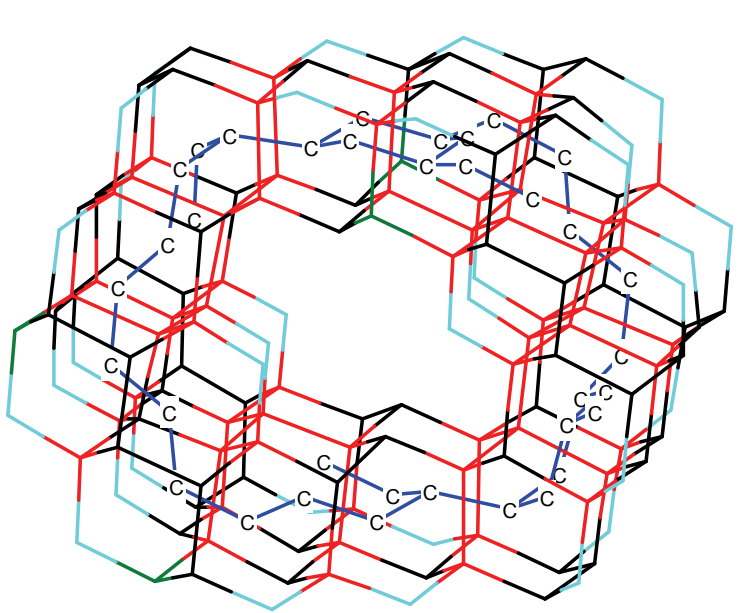

Figure 19. Achiral tunnel similar to the coronamantane-6666, with the same color code. Only part of dualist vertices and edges appears. Color code as in Figures 15-18.

If one drills a straight cut for overlappping pairs of adjacent carbon atoms into this lattice, one obtains an achiral tunnel represented in Figure 19, similar to the coronamantane of Figure 16. By analogy, it is possible to have achiral tunnels similar to coronamantanes 5555 , 7777 , and 5757.

Based on the chirality of [123]tetramantane, one can devise helical polymantanes such as [12341234]nonamantane (Figure 20) where the repeating pattern conserves the same chirality.

If from the multi-tier adamantane lattice one cuts a helical tunnel modeled after Figure 20, then the result is a chiral hole that may lead to practical applications, for instance hosting simple achiral reactants that would afford chiral reaction products. In Figure 21 one can see the tunnel in the three-tier portion of the diamond lattice with the same color code as in Figures 15-19.

In Figure 22 one can see the shape of the circular dualist surrounding the chiral tunnel so that one may compare it with the achiral coronamantane-6666 (Figure

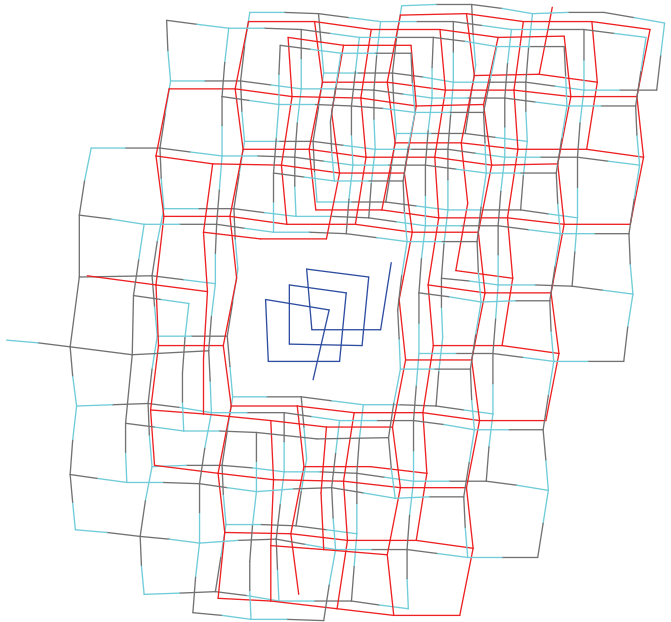

Figure 21. The result of carving out a helical [1234123412341]tetradecamantane (but not its dualist) from the three-tier diamond lattice.

16) and the related achiral tunnel shown in Figure. It is evident that achiral coronamantanes and tunnels have internal mirror symmetry, whereas no such symmetry exists in the chiral dualists, although their numbers of vertices are the same.

There is a rich bibliography about zeolites having chiral cavities and tunnels with corresponding applications. ${ }^{26-28}$ Although till now coronamantanes and tunnels in the diamond lattice have not been obtained experimentally, they should be considered as worthy challenges for the future.

\section{CONCLUSION}

Awareness about diamondoids has increased lately, ${ }^{29,30}$ but till now little has been published about holes and tunnels in diamondoids. ${ }^{1}$ The present paper examined various possibilities of having diamondoids with holes, i.e. small portions of the diamond lattice carbon atoms

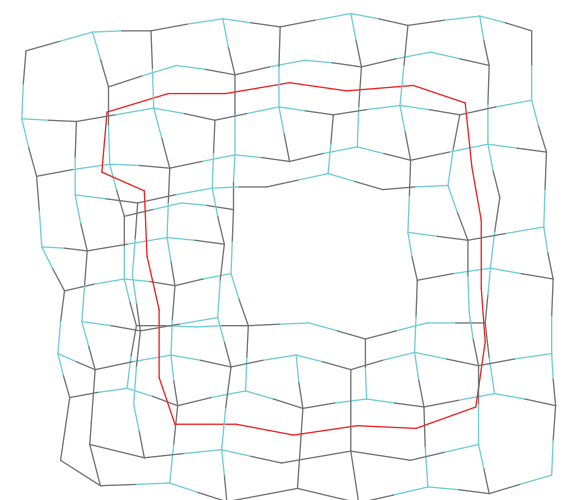

Figure 22. The circular dualist closest to the chiral tunnel shown in Figure 21. Color code as in Figures 16-21. 
with outer and inner dangling bonds connected to hydrogen atoms. Two types of molecular coronamantanes have been examined, namely those with buried holes in the diamondoid (analogous to closed pores in polymer foams) and those with holes open to external space analogous to open pores in polymer foams. The most interesting latter ones have dualists that are quasi-flat being analogs of perhydrocoronoids. Chiral and achiral holes and tunnels in such diamondoids may lead to interesting applications. On replacing certain inner $\mathrm{CH}$ groups by $\mathrm{N}$ or $\mathrm{P}$ atoms, or inner $\mathrm{CH}_{2}$ groups by $\mathrm{O}$ or $\mathrm{S}$ atoms, conditions would be created for coordinating metallic atoms or cations, perhaps selectively, according to the size and geometry of the cavity and the nature of the heteroatom.

\section{REFERENCES}

1. A. T. Balaban, D. J. Klein, and W. A. Seitz, Internat. J. Quantum Chem. 60 (1996) 1065-1068.

2. (a) A. Tomita and Y. Tamai, J. Catal. 27 (1972) 293-300; (b) A. Tomita and Y. Tamai, J. Phys. Chem. 78 (1974) 2254-2258.

3. L. Ci, L. Song, D. Jariwala, A. L. Elias, W. Gao, M. Terrones, and P. M. Ajayan, Adv. Mater. 21 (2009) 4487-4491.

4. M. Lukas, V. Meded, A. Vijayaraghavan, L. Song, P. M. Ajayan, K. Fink, W. Wenzel, and R. Krupke, Nature Commun. 4 (2013) $1-7$.

5. W. Jenny and R. Peter, Angew. Chem. Int. Ed. Engl. 4 (1965) 979.

6. (a) S. V. Cyvin, J. Brunvoll, and B. N. Cyvin, Theory of Coronoid Hydrocarbons. Springer, Berlin, 1991; (b) S. V. Cyvin, J. Brunvoll, B. N. Cyvin, J. L. Bergan, and E. Brensdal, Struct. Chem. 2 (1991) 555-566.

7. A. T. Balaban, J. Brunvoll, J. Cioslowski, B. N. Cyvin, S. J. Cyvin, I. Gutman, He Wenchen, He Wenjie, J.V. Knop, M. Kovacević, W.R. Müller, K. Szymanski, R. Tosić, and N. Trinajstić, Z. Naturforsch. 42c (1987) 863-870.

8. (a) A.T. Balaban, Pure Appl. Chem, 54 (1982) 1075-1096; (b) A. T. Balaban, Rev. Roum. Chim., 33 (1988) 699-707.

9. A. T. Balaban and M. Randić, J. Chem. Inf. Comput. Sci. 44 (2004) 50-59.
10. (a) D. J. Klein and L. Bytautas, J. Phys. Chem. A, 103 (1999) 5196-5210; (b) O. Ivanciuc, L. Bytautas, and D. J. Klein, J. Chem. Phys. 116 (2002) 4736-4748; (c) O. Ivanciuc, D. J. Klein, and L. Bytautas, Carbon 40 (2002) 2063-2083.

11. D. J. Klein, Pure Appl. Chem. 80 (2008) 1399-1414.

12. F. Diederich and H. A. Staab, Angew. Chem. Int. Ed. Engl. 17 (1978) 372-374

13. H. A. Staab and F. Diederich, Eur. J. Inorg. Chem. 116 (1983) 3487-3503.

14. A. R. Katritzky and C. M. Marson, J. Am. Chem. Soc. 105 (1983) 3279-3285.

15. A. K. Geim and K. S. Novoselov, Nature Materials 6 (2007) 183-191.

16. D. J. Klein and A. T. Balaban, The Open Org. Chem. J. 5 (2011) 27-61.

17. A. T. Balaban and K. P. C. Vollhardt, The Open Org. Chem. J. 5 (2011) 117-126.

18. H. Liu and D. S. Dandy, Diamond Chemical Vapor Deposition: Nucleation and Early Growth Stages (Materials Science and Process Technology) Noyes Publication, 1997.

19. K. V. Ravi, Synthetic Diamond-Emerging CVD Science and Technology (K. E. Spear, J. P. Dismukes, eds.), Wiley, New York, 1994, pp. 533-580.

20. G. Davies, Diamond, Adam Hilger, Bristol, UK, 1984.

21. A. T. Balaban and P. V. R. Schleyer, Tetrahedron 34 (1978) 3599-3609.

22. A. T. Balaban and C. Rücker, J. Chem. Inf. Comput. Sci. 41 (2001) 1145-1149.

23. (a) A. T. Balaban, K. B. Chilakamarri, and D. J. Klein, J. Math. Chem. 45 (2009) 725-747; (b) A. T. Balaban, MATCH, Commun. Math. Comput. Chem. 66 (2011) 139-162.

24. A. T. Balaban, J. Chem. Inf. Model. 52 (2012) 2856-2869.

25. A. T. Balaban, J. Math. Chem. 51 (2013) 1043-1055.

26. Y. Li , J. Yu, Z. Wang, J. Zhang, M. Guo, and R. Xu, Chem. Mater. 17 (2005) 4399-4405.

27. J. M. Castillo, T. J. H. Vlugt, D. Dubbeldam, S. Hamad, and S. Calero, J. Phys. Chem. C 114 (2010) 22207-22213.

28. J. Jiang, R. Babarao, and Z. Hu, Chem. Soc. Rev. 40 (2011) 3599-3612.

29. G. A. Mansoori, P. L. B. de Araujo, and E. S. de Araujo, Diamondoid Molecules: With Applications in Biomedicine, Materials Science, Nanotechnology and Petroleum Science. World Scientific Publ., New York, 2012.

30. A. T. Balaban, Diamond and Related Nanostructures (Eds.: M. V. Diudea, C. L. Nagy), Springer, Dordrecht, 2013, pp. 1-27. 